\section{Temperature and brain death determination: need for updated criteria}

\section{Michael A. Meyer}

Clinical Neurology and Nuclear Medicine, University of New York, Buffalo, NY, USA

As noted by Greer and colleagues, ${ }^{1}$ the birth of the brain death concept dates back to the 1959 report by Mollaret and Goulon on 23 comatose patients. ${ }^{2}$ Since that time, new and better criteria for the determination of brain death have developed, as well new technologies that assess brain function, including the use PET imaging to confirm the clinical diagnosis for brain death. ${ }^{3}$ Although other new ancillary tests have developed that also include CT Angiography, there remains considerable variability in the clinical protocol checklists at individual medical centers on how the final diagnosis of brain death is achieved. Significant discrepancies were present among 38 medical centers studied by Greer and colleagues $^{1}$ for brain death guideline performance, preclinical testing, clinical examination, apnea testing, and ancillary tests.

Although variability can be partly accounted for the fact that some clinical exam tests for brain death require subjective evaluation and individual physician judgment, an absolute measurement that showed surprising variability amongst the centers studied was the minimum core temperature for brain death determination. As shown by Greer and colleagues, ${ }^{1}$ almost $75 \%$ of all centers placed the minimum temperature at about $32^{\circ} \mathrm{C}$. Since even mild therapeutic hypothermia clinical protocols have a target core temperature of $33^{\circ} \mathrm{C}$ for the production of a medically induced comatose state,${ }^{4}$ it seems paradoxical that brain death protocols would find one degree lower at $32{ }^{\circ} \mathrm{C}$ to be an acceptable minimum to conduct a clinical exam for brain viability. Furthermore, the minimum temperature for apnea testing was set at $36.5^{\circ} \mathrm{C}$ by $70 \%$ of all 38 US medical cen- ters sampled. ${ }^{4}$ Chin and colleagues ${ }^{5}$ studied this variability amongst 36 physicians in Singapore, and noted that a cumulative total of $79 \%$ of all physicians set a lower limit of $35^{\circ} \mathrm{C}$ as a required precondition for brain death determination - only $6 \%$ of the group found a temperature below $34^{\circ} \mathrm{C}$ as acceptable minimum temperatures. Therefore, the proposed new guidelines by Wijdicks and colleagues ${ }^{6}$ with regards to temperature are very reasonable: warming blankets should be used to achieve a normal or near normal body temperature at being above $36^{\circ} \mathrm{C}$.

As these updated guidelines are relatively new, US hospitals and State Departments of Health should correspondingly update their criteria for brain death to include this minimum temperature criteria as being $36^{\circ} \mathrm{C}$. For example, multiple hospitals within the State of New York are still following the December 2005 Guidelines for Determining Brain Death from the NY State Dept. of Health, which has a checklist that requires core body temperature to be $32^{\circ} \mathrm{C}$ or above $\left(89.6^{\circ} \mathrm{F}\right)$. This level may be considered by others to be too low to conduct a valid brain death exam, and all hospitals should rapidly update and revise their criteria according to Wijdicks and colleagues. ${ }^{6}$

For an excellent review on the diagnosis of brain death, the interested reader is directed to the review of Machado ${ }^{7}$ appearing in this journal; the author reviews all aspects of brain death and cites nine different references where the minimum temperature for brain death exams appear to have been at least $32.2^{\circ} \mathrm{C}$. Given the new data listed above, it is clearly time for a reconsideration of the how we approach the exam for diagnosis of brain death - normal or near normal temperatures of $36^{\circ} \mathrm{C}$ and above are very reasonable starting points.

\section{References}

1. Greer DM, Varelas PN, Haque S, Wijdicks EF. Variability of brain death determina-
Correspondence: Michael A. Meyer, Professor of Clinical Neurology and Nuclear Medicine, University of New York, Buffalo, NY, USA. E-mail: michaelandrewmeyer@yahoo.com

Key words: brain death, temperature, coma.

Received for publication: 24 August 2010. Accepted for publication: 24 August 2010.

This work is licensed under a Creative Commons Attribution 3.0 License (by-nc 3.0).

CCopyright M.A. Meyer, 2010

Licensee PAGEPress, Italy

Neurology International 2010; 2:e15

doi:10.4081/ni.2010.e15

tion guidelines in leading US neurologic institutions. Neurology 200870:284-9.

2. Mollaret P, Goulon M. Le Coma Depasse (preliminary memoir). Rev Neurol (Paris). 1959 Jul;101:3-15.

3. Meyer MA. Evaluating brain death with positron emission tomography: case report on dynamic imaging of 18F-fluorodeoxyglucose activity after intravenous bolus injection. J Neuroimaging 1996;6: 117-9.

4. Jehle D, Meyer M, Gemme S. Beneficial response to mild therapeutic hypothermia for comatose survivors of near-hanging. Am J Emerg Med 2010;28:390.e1-3.

5. Chin KJ, Kwek TK, Lew TW. A survey of brain death certification - an impetus for standardisation and improvement Ann Ann Acad Med Singapore 2007;36:987-9.

6. Wijdicks EF, Varelas PN, Gronseth GS, Greer DM. American Academy of Neurology. Evidence-based guideline update: determining brain death in adults: report of the Quality Standards Subcommittee of the American Academy of Neurology. Neurology 20108;74:1911-8.

7. Machado C. Diagnosis of brain death. Neurol Int 2010:2(e2). 